\title{
La actividad física y la recreación como estrategias para impactar a las comunidades locales y promover el desarrollo de habilidades blandas en el estudiantado: la experiencia de extensión universitaria con estudiantes de diferentes carreras en la Sede Interuniversitaria de Alajuela, Costa Rica
}

Physical Activity and Recreation as Strategies to Impact

Local Communities and Promote the Development of Soft Skills in Students: The Experience of University Extension with Students From Different Careers at the Interuniversity Campus of Alajuela, Costa Rica

Yanixa Miranda-Benavides Universidad Nacional Costa Rica

yanixa.miranda.benavides@una.cr D https://orcid.org/0000-0002-8133-8497

Karla Miranda-Benavides Universidad Nacional Costa Rica

karla.miranda.benavides@una.cr https://orcid.org/0000-0002-5862-492X

Gaudy Prendas-Aguilar Universidad Nacional Costa Rica 
Revista Universidad En DiÁlogo • Vol. 11, N. ${ }^{\circ}$ 1, Enero-Junio, 2021 • 135-155

ISSN 2215-2849 • EISSN: 2215-4752

URL: http://www.revistas.una.ac.cr/index.php/dialogo/index CorReo ElECTRÓNICO: universidadendialogo@una.cr DOI: http://doi.org/10.15359/udre.11-1.5

Resumen. Este artículo presenta las experiencias de la población estudiantil de diferentes carreras de la Sede Interuniversitaria de Alajuela de la Universidad Nacional, que, como parte de su proceso de aprendizaje en el curso Actividades y Eventos Recreativos, diseña y ejecuta actividades que promueven la actividad física y la recreación con diferentes poblaciones de la provincia de Alajuela. Para la sistematización de esta experiencia se aplicó un cuestionario al estudiantado y a las personas responsables de las organizaciones donde se efectuaron las actividades, con el propósito de conocer su percepción en relación con los resultados obtenidos. Este ejercicio es una prueba de cómo por medio del aprendizaje y de la extensión es posible fortalecer habilidades en el estudiantado e impactar a las comunidades.

Palabras clave: actividad física, extensión universitaria, habilidades blandas.

Abstract: This article discloses situations students from different careers of the National University, Alajuela Interuniversity Campus, have experienced. As part of their learning process in the course "Actividades y Eventos Recreativos" (Activities and Recreational Events), they designed and practiced actions that promote physical and recreational activities in different communities in Alajuela, one of the seven provinces of Costa Rica. This exercise proves that it is possible to reinforce students' abilities and impact local communities through learning and extension programs. For the systematization of this experience, the students and people in charge of organizations where the activities took place were asked to fill out a questionnaire to know their perception regarding the obtained results.

Keywords: physical activity, soft skills, university extension.

\section{Introducción}

La promoción de estilos de vida saludables en las personas es una tarea pendiente en Costa Rica; por lo tanto, las acciones que se realizan para el fomento de actividades que traen mejoras a la salud, como la recreación y el deporte, representan un impacto directo en varias direcciones: por un lado, en las poblaciones beneficiadas con los eventos organizados y, por otra parte, en el estudiantado, conformado por profesionales integrales, comprometidos con el bienestar de los individuos y de la sociedad.

Este trabajo surge como una acción académica del curso llamado Actividades y Eventos Recreativos, un curso optativo que se imparte en la Sede Interuniversitaria de Alajuela y que tiene como característica particular la matrícula de estudiantes de múltiples disciplinas, que incluye las áreas de Administración, Inglés, Ingeniería en Sistemas y Química Industrial. 
Esta variedad de disciplinas genera una sinergia muy particular en el curso, que a su vez se traslada a las propuestas de diseño de cada actividad que organizan los grupos de trabajo (conformados por cuatro o cinco estudiantes), quienes diseñan y aplican una actividad recreativa en una comunidad, que el equipo define de acuerdo con su afinidad y cercanía con la población seleccionada.

En este artículo se presentan los resultados de la experiencia estudiantil en la planeación y organización de esta actividad, así como el impacto en el desarrollo de habilidades de los y las estudiantes. Además, se muestran los resultados de la percepción de las organizaciones y poblaciones beneficiadas; esta es una iniciativa en la que se traslada la experiencia del aula hacia los entornos de la universidad, con el fin de promover un impacto en la población de la provincia donde se ubica el campus universitario.

Con la ejecución de actividades de esta naturaleza fácilmente se puede reconocer el beneficio que reciben las comunidades, las instituciones y los futuros profesionales, quienes trasladan a su vida profesional el compromiso con la sociedad, que a su vez les permite crecer en la formación de habilidades para la vida.

\section{Antecedentes y base teórica}

Actualmente, el estilo de vida de las personas muestra una deficiencia en los hábitos para mejorar su salud, lo que aumenta la propensión a enfermedades y un estado de salud debilitado. La limitación de acceso a espacios públicos y las condiciones sociales, económicas y culturales generan un vacío en la práctica de hábitos promotores de la salud, y es en este aspecto donde se identifica la necesidad de generar procesos educativos que motiven en el estudiantado el reconocimiento y la trascendencia del cuidado de la salud, con el fin de impactar a las personas más cercanas a él y a sus comunidades, por medio de la actividad física, el deporte y la recreación, con el fin de expandir el espacio académico al entorno, para vincular a la población desde la realidad de cada persona.

Con el fin de combatir los alarmantes datos obtenidos en relación con el estado de salud de los y las costarricenses, que evidencian un aumento sustancial de factores de riesgo asociados a problemas de salud, tales como los mencionados en el último informe El estado de la seguridad alimentaria y la nutrición en el mundo 2017, y la Encuesta Actualidades del 2016 de la Universidad de Costa Rica, citada por Salas (2017). En los cuales se menciona el incremento del sedentarismo y de la obesidad en la población, lo que genera un foco de atención para tomar acciones desde el ámbito universitario y las entidades de salud. 
Revista Universidad en Diálogo • Vol. 11, N. ${ }^{\circ}$ 1, Enero-Junio, 2021 • 135-155

ISSN 2215-2849 • EISSN: 2215-4752

URL: http://www.revistas.una.ac.cr/index.php/dialogo/index CorReo ElECTRÓNICO: universidadendialogo@una.cr DOI: http://doi.org/10.15359/udre.11-1.5

Estos datos afirman la necesidad de desarrollar, desde los espacios académicos, acciones que promuevan un cambio en las conductas sedentarias de las diferentes poblaciones y comunidades, impactando de forma positiva por medio de acciones a favor de la actividad física y la recreación. Además, educando a profesionales con una formación integral, con una mayor conciencia sobre la importancia de cuidar su salud y prevenir una serie de afectaciones a corto y largo plazo.

Salud es un término con una serie de definiciones que se mantienen en constante evolución, tal como lo mencionan Santamaría y Martínez (2018), quienes definen la salud "como un estado del cuerpo que se caracteriza por preservar las funciones (físicas y psíquicas) del organismo a un nivel adecuado, así como de mantener un grado de desarrollo social, que complete el bienestar personal del individuo" (p. 8).

Los estilos de vida saludables inician desde la educación para la salud en los diferentes espacios de acción, esta se caracteriza, además, por constituir un conjunto de aprendizajes estructurados desde la formación de hábitos, comportamientos saludables, procesos informativos y actitudes positivas, con el fin de establecer decisiones responsables para el cuidado del cuerpo (Guerrero, Jiménez y López, 2013).

Los cambios culturales y sociales marcan una pauta de comportamiento en la sociedad. Así lo mencionan Moscoso, Sánchez, Martin y Pedrajas (2014): "La difusión de hábitos y comportamientos sedentarios plantea uno de los más serios problemas para las sociedades contemporáneas" (p. 79.). Los avances en tecnología, salud, alimentación, transporte y formas de entretenimiento han generado una serie de factores que influencian a la población a desarrollar hábitos cada vez más sedentarios y que afectan su salud, lo cual aumenta los factores de riesgo para la aparición de enfermedades prevenibles relacionadas con hábitos perjudiciales.

Las nuevas tendencias educativas apuntan a un desarrollo de modelos educativos que logren fomentar un desarrollo de gestión que permita fortalecer los centros educativos, y así establecer un engranaje de los factores internos y externos que interfieren en los espacios educativos y se relacionan con las dinámicas de los mismos. Estos poseen diferencias sustanciales en relación con las empresas tradicionales.

Chacón (2014) menciona que la gestión busca un desarrollo de competencias y saberes que pretende ir más allá de procedimientos individualizados, para así lograr el desarrollo de conocimientos con el aporte de un trabajo en equipo y la resolución de situaciones que busquen solventar las demandas 
y necesidades de la sociedad actual. Esto último se vincula estrechamente con una de las principales habilidades que solicitan las personas empleadoras, como la capacidad para resolver problemas.

Ramírez, Calderón y Castaño (2015) recalcan que existen una serie de cambios del entorno relacionados con las exigencias que la sociedad brinda a las organizaciones, que han provocado una serie de transformaciones hacia la apertura y el dinamismo, por lo cual no solo se requieren los procesos administrativos base, sino que deben incluirse principios como la planeación, la comunicación, la solución a los problemas y la toma de decisiones, siendo estos parte vital para la dirección y los procesos de liderazgo que buscan el alcance de metas y objetivos.

La incorporación de nuevas estrategias o proyectos debe visualizarse como una oportunidad de cambio y adaptación al entorno, no como una amenaza del mismo. La estructuración de nuevos sistemas ayuda a mantenerse acorde con la realidad y salir de mecanismos obsoletos, no obstante, debe generarse un cambio ordenado que ayude a mejorar la dinámica del centro y no genere tanta resistencia de parte de los miembros de la institución.

En cuanto a los procesos de gestión de proyectos, Pérez (2013) establece que buscan la asociación de múltiples factores con el fin de garantizar los propósitos educativos en el desarrollo de las instituciones. Cada proyecto educativo planteado debe responder a necesidades determinadas en diferentes campos de acción, que varían según lo planteado por la institución. De ahí la importancia de generar sistemas de elaboración de proyectos acordes con las necesidades inmediatas y futuras, tomando en cuenta las características propias del centro educativo y su entorno. Para ello, la utilización de los principios administrativos y las estrategias de elaboración de proyectos permitirá desarrollar los campos de acción claros para disminuir los factores de riesgo en su implementación.

Existen oportunidades de cambio en aspectos socioculturales que justifican el aumento de peso como parte del estilo de vida, un mayor uso de la tecnología, que hace personas más sedentarias, y el acceso a alimentos procesados, que afectan la salud de todas las personas; hábitos que han ocasionado una menor calidad de vida para la ciudadanía costarricense.

Las acciones que buscan estilos de vida saludables requieren una sensibilización de la población definida, donde se trate como un asunto de prevención que las personas integren en su vida diaria la actividad física como parte de su rutina, y una manera de lograrlo es a través de iniciativas grupales para la 
Revista Universidad en Diálogo • Vol. 11, N. ${ }^{\circ}$ 1, Enero-Junio, 2021 • 135-155

ISSN 2215-2849 • EISSN: 2215-4752

URL: http://www.revistas.una.ac.cr/index.php/dialogo/index CorReo ElECTRÓNICO: universidadendialogo@una.cr DOI: http://doi.org/10.15359/udre.11-1.5

integración del estudiantado con diferentes grupos de población que, según sus características, fortalezas y debilidades, puedan identificar en el deporte y las actividades recreativas un método de diversión y una forma de llevar salud a sus vidas y a la de terceras personas.

\section{¿Por qué es importante el desarrollo de habilidades blandas?}

Las habilidades blandas son aquellas aptitudes y actitudes que le permiten a una persona actuar de manera efectiva. Consisten en una serie de destrezas que incluyen el pensamiento crítico, la ética y la posibilidad de adaptarse al cambio. De acuerdo con Vargas (2017), las personas trabajadoras deben ejercer una serie de habilidades para cumplir sus tareas y alcanzar sus objetivos. Dentro de estas habilidades, hay dos tipos: "Las duras, enfocadas en evaluar el coeficiente intelectual y las capacidades técnicas individuales, llamadas blandas, que se enfocan, a la persona con su entorno" (p. 14).

Se habla de que, a través del tiempo, las habilidades blandas permiten promover entre las personas una fuerte ética laboral, una actitud positiva, el trabajo en equipo, el trabajo bajo presión y la flexibilidad. Dentro de las habilidades blandas se muestran tres grandes grupos, según Vargas (2017), el primero enfocado en la conducta, el segundo contiene a las habilidades básicas o esenciales y en el tercer grupo se encuentran las habilidades técnicas o funcionales específicas de la descripción del cargo a desempeñar.

Es necesario que el estudiantado atienda las exigencias profesionales actuales, desarrollando una serie de habilidades que le permitan una comunicación fluida, madurez para la toma de decisiones y la resolución de problemas, con una actitud crítica, con un alto grado de creatividad y con la capacidad de enfrentar situaciones que se pueden presentar en su desarrollo e integración laboral.

Una de las principales redes sociales utilizadas en la actualidad para formar redes comerciales y profesionales es la conocida como Linkedin, a través de esta se efectúan transacciones entre empresas y personas, abriendo un gran mercado en la gestión del talento humano, lo que permite conexiones rápidas en tiempo real y posibilita la ubicación de las personas en puestos de trabajo acordes con sus habilidades, experiencia y formación académica. 
El tema de habilidades es mencionado con mucha frecuencia por profesionales del área de talento humano, y esta red social no es la excepción. En una publicación del 14 de noviembre del año 2018, referente a las diez habilidades blandas más solicitadas en el mercado laboral, se indica que las personas empleadoras buscan en los perfiles de las personas candidatas que evalúan para sus puestos de trabajo las siguientes habilidades: buena comunicación, buena organización, trabajo en equipo, puntualidad, pensamiento crítico, sociabilidad, creatividad, habilidades interpersonales de comunicación y facilidad de adaptación.

Por otra parte, Neumann (2015) cita que es necesario desde los contextos educativos desarrollar estas habilidades, enfocados en potenciar tres habilidades clave: el autoconocimiento, reconocer fortalezas y debilidades y enfocarlas a favor de la organización; la empatía, la cual busca asimilar y entender el mundo de otras personas, sin prejuicios; y por último el liderazgo, entendido como la capacidad de provocar un rendimiento extraordinario y coordinado en su equipo de trabajo.

En las organizaciones existe una escasez de personal que posea las habilidades mencionadas anteriormente, siendo que, en muchas ocasiones, las personas se enfocan en su desarrollo académico, y en la actualidad las empresas se enfocan en la búsqueda de personas con potenciales socioemocionales. Si se favorecen estas competencias desde el aula, se podrán formar profesionales integrales, con cualidades para ser mejores trabajadores y trabajadoras en su entorno laboral.

Es por esto que este tipo de experiencia en el estudiantado permite construir, a través de un contacto con la fuente primaria, la necesidad de fomentar la actividad física dentro de diferentes poblaciones. Por medio de la utilización de un modelo de acción que le permite al estudiantado el desarrollo y la puesta en práctica de una actividad, donde se benefician las comunidades locales que reciben el taller y, a su vez, las personas estudiantes con la generación y el desarrollo de diferentes habilidades.

Con base en el modelo de competencias propuesto por Werther y Davis en su libro Administración de recursos humanos. Gestión de capital humano se muestra a continuación la Figura 1: 


\section{Figura 1}

Modelo de gestión de competencias

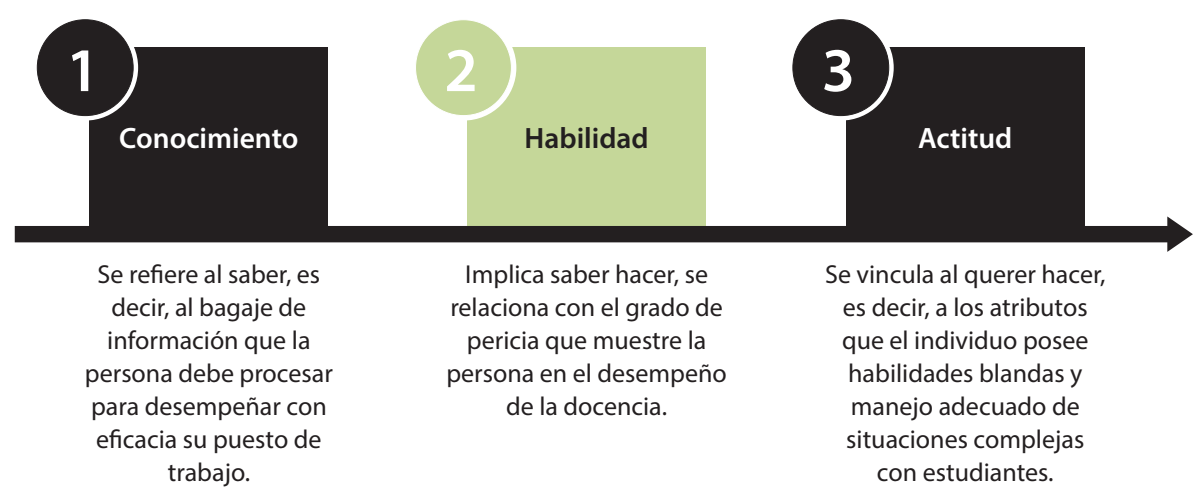

Nota. elaboración propia con datos tomados de Werther, W, Davis, K, 2014, p. 143.

Este modelo permite alinear la metodología utilizada en el curso Actividades y Eventos Recreativos, en el cual se articulan los siguientes aspectos:

- La sensibilización del estudiantado sobre la importancia del mejoramiento de la salud y la calidad de vida de las personas, por medio de la actividad física

- Detección de necesidades específicas en las comunidades locales, en las cuales se puede crear un impacto positivo.

- Aprendizaje y puesta en acción de actividades y talleres, que le permiten al estudiantado transmitir los conocimientos adquiridos en el curso universitario.

- Generación de habilidades blandas y competencias socioemocionales necesarias para su desempeño como individuos sociales y personas profesionales

- Motivación y actitud de cambio en las personas, con lo que se promueve el desarrollo de las comunidades y poblaciones involucradas.

- Fomento del pensamiento crítico, la proactividad y el análisis continuo de las necesidades del medio del que forman parte las personas. 
URL: http://www.revistas.una.ac.cr/index.php/dialogo/index

CORREO ELECTRÓNICO: universidadendialogo@una.cr

DOI: http://doi.org/10.15359/udre.11-1.5

\section{Metodología}

La investigación se enfoca en el paradigma cualitativo, que busca un abordaje desde la realidad de cada persona, para interpretar fenómenos y experiencias estableciendo cómo estas influyen en cada quien y su entorno. Se trata al final de identificar la naturaleza profunda de las realidades, su sistema de relaciones, su estructura dinámica, con el claro propósito de proveer descripciones detalladas de los contextos estudiados; se debe hablar de un entendimiento en profundidad, en lugar de exactitud.

\section{Participantes}

Para esta investigación, se trabajó con una población de cuarenta y cuatro personas estudiantes de la Universidad Nacional, matriculadas en diferentes carreras en la Sede Interuniversitaria de Alajuela, inscritas en el curso Administración del Tiempo y Programación de Eventos Recreativos, quienes trabajaron directamente con personas de diferentes comunidades $\mathrm{e}$ instituciones de la provincia de Alajuela, tanto en el ámbito privado como en el público, y de diferentes rangos etarios.

\section{Técnicas e instrumentos de recolección de datos}

\section{Observación}

Se utiliza la técnica de observación, la cual, según Campos, Covarrubias y Lule (2012), es la forma más sistematizada y lógica para el registro visual y verificable de lo que se pretende conocer, es captar de la manera más objetiva posible lo que ocurre en el mundo real, ya sea para describirlo, analizarlo o explicarlo desde una perspectiva científica.

De la observación se derivan varios tipos; para esta investigación se utilizaron dos de estos, los cuales son mencionados por González y Orozco (2013):

- Observación no participante: Es realizada por agentes externos que no tienen intervención alguna dentro de los hechos; por lo tanto, no existe relación con sujetos del escenario; tan solo se es espectador o espectadora de lo que ocurre, y quien investiga se limita a tomar nota de lo que sucede para conseguir sus fines.

- Observación participante: La persona investigadora se involucra en los procesos de quienes observa, y es plenamente aceptada, por tanto, se estima que lo observado no se ve afectado por la acción de quien observa. 


\section{Cuestionario}

Según Ramos (2014), el cuestionario es el instrumento que busca la recolección de datos destinados a obtener las respuestas sobre la temática a consultar, obteniendo las impresiones de las personas participantes. Para esta investigación se establecieron dos cuestionarios, uno enfocado en el estudiantado y el otro en la población administrativa:

1. El primer cuestionario está dirigido a las organizaciones y busca recopilar las opiniones de las personas responsables de las instituciones sobre la implementación de este tipo de actividades y su impacto en la población.

2. El segundo cuestionario está dirigido a la población inscrita en el curso, para conocer su percepción de la aplicación de la actividad y la experiencia vivida con la ejecución de esta.

\section{Procedimientos}

Para la ejecución de la propuesta se estableció como metodología evaluativa del curso la preparación de una actividad recreativa dirigida a diferentes poblaciones, con el fin de aplicar los contenidos y beneficiar a la población de la provincia. Para esto, se dividió al grupo de personas estudiantes en subgrupos de trabajo, como parte del proyecto final, el cual se realiza con una población definida, según el interés del estudiantado.

Posteriormente, se solicitan los permisos respectivos y se realiza un diagnóstico sobre la población seleccionada. A partir de los resultados obtenidos, se efectúa un plan de trabajo enfocado en el desarrollo y la promoción de la salud acorde con las necesidades de la institución y la población beneficiada.

Para cada uno de los procesos se realizó un acompañamiento permanente por parte del profesor del curso, quien brindó los parámetros básicos en relación con el diagnóstico de la institución y la población; también se desarrolló una estructura general para la programación y ejecución de las propuestas. Una vez efectuado el diagnóstico, los grupos de trabajo presentan los resultados de las actividades realizadas y sus experiencias a los miembros del grupo.

\section{Análisis de datos}

Como parte del análisis de datos, se efectúa una recopilación de los resultados de los cuestionarios aplicados a las personas encargadas del área administrativa 
URL: http://www.revistas.una.ac.cr/index.php/dialogo/index

CORREO ELECTRÓNICO: universidadendialogo@una.cr

DOI: http://doi.org/10.15359/udre.11-1.5

de los centros en los que se llevaron a cabo las diferentes actividades recreativas y a la población estudiantil.

Se establece una presentación sobre las percepciones y opiniones de las personas participantes directas e indirectas, así como la observación no participante de personas docentes externas en las presentaciones de los proyectos, con el fin de evaluar el impacto que posee este tipo de propuestas evaluativas en las poblaciones involucradas.En la Figura 2 se muestra la ubicación geográfica de las instituciones y empresas participantes en esta acción de extensión universitaria:

Figura 2

Ubicación geográfica de las instituciones y empresas

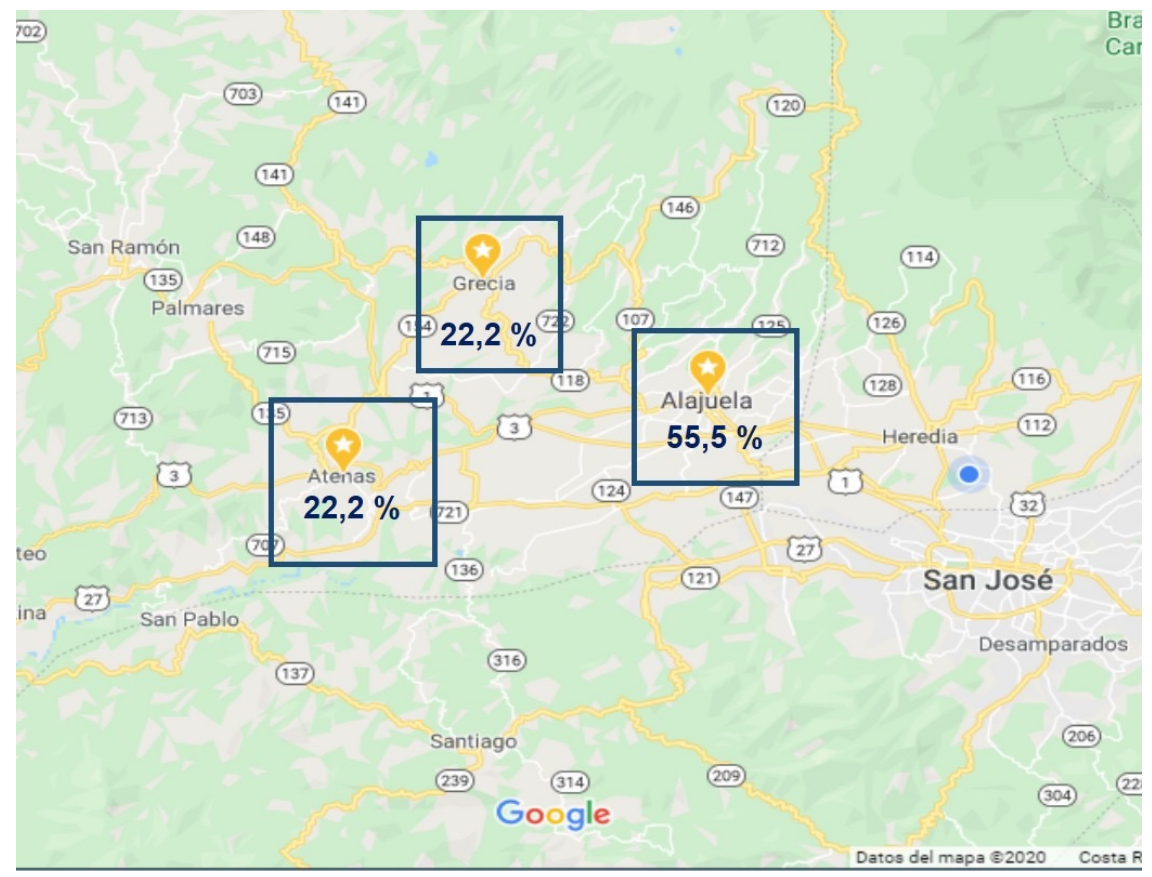

Nota. Imagen tomada de https://www.google.com/maps/. Datos de elaboración propia

Según el interés y la viabilidad de las instituciones elegidas por las personas estudiantes para llevar a cabo las actividades, los cantones más cercanos en ubicación a la Sede Interuniversitaria de Alajuela fueron los más recurrentes, con más de un $50 \%$ de las acciones ejecutadas, y el restante $45 \%$ divididas en los cantones de Atenas y Grecia. 


\section{Figura 3}

Naturaleza de las organizaciones

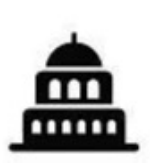

$\mathbf{5 5 , 5 \%}$

Pública

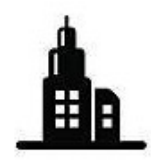

33,3\%

Privada

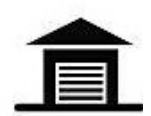

$11,1 \%$

Comunal

Nota. imágenes tomadas de google.

com. Datos de elaboración propia.

Más de la mitad de las organizaciones visitadas por el estudiantado fueron del sector público, esto evidencia la apertura que existe de este tipo de instituciones para el abordaje de acciones que ayuden al mejoramiento de la salud de las personas; sin embargo, el sector privado presentó una recepción del 33\%, lo que también se presenta como un dato bastante positivo; no obstante, es importante incrementar actividades en un futuro proyecto. Por último, existe una única organización de naturaleza comunal, con un $11 \%$.

\section{Figura 4}

Experiencia y relevancia de la actividad recreativa desarrollada

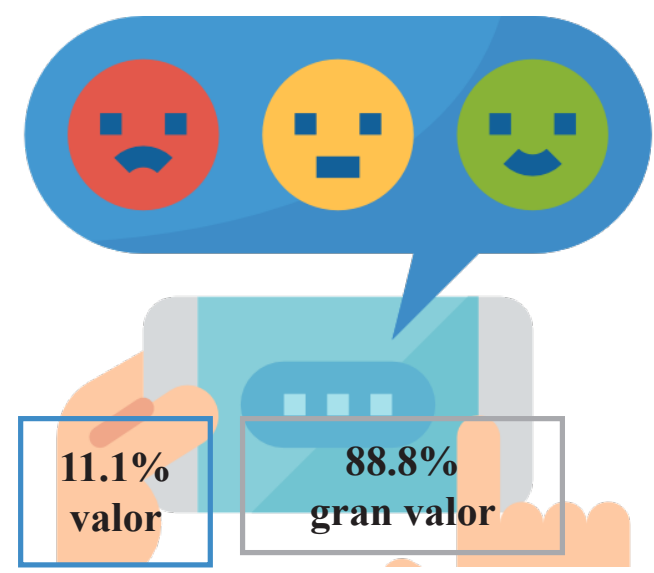

Nota. iconos de https://www.flaticon.com/.

Datos de elaboración propia. 
URL: http://www.revistas.una.ac.cr/index.php/dialogo/index

CORREO ELECTRÓNICO: universidadendialogo@una.cr

DOI: http://doi.org/10.15359/udre.11-1.5

Según la evaluación efectuada por las personas representantes de las instituciones, las actividades diseñadas por el estudiantado causaron un impacto muy positivo, siendo calificadas como de gran valor en un $88 \%$.

\section{Figura 5}

Tipos de habilidades desarrolladas por el estudiantado

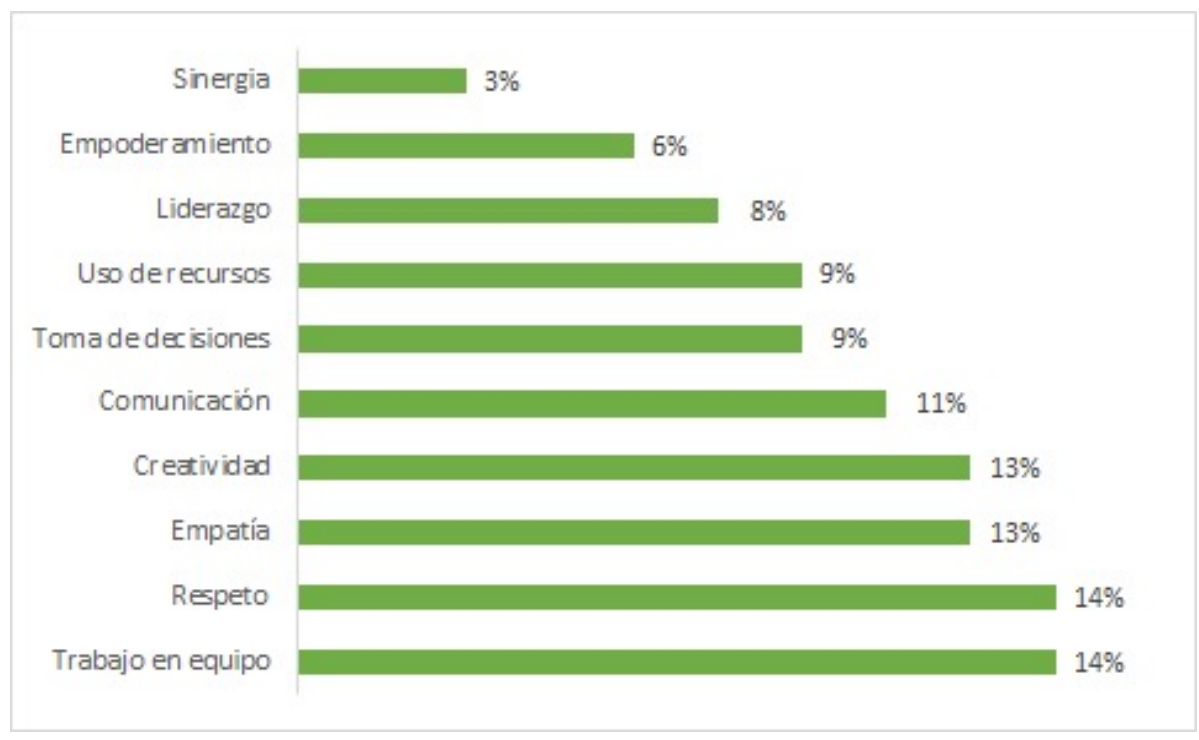

Nota. Elaboración propia

De los tipos de habilidades que mostraron las personas estudiantes en el desarrollo de las actividades presentadas, las que fueron de mayor relevancia para las personas representantes de las organizaciones fueron el trabajo en equipo y el respeto, cada una con un $14 \%$, seguidas por la empatía y la creatividad con un $13 \%$, además de la comunicación con un $11 \%$. De menor relevancia fueron evaluadas habilidades como la toma de decisiones, el uso de recursos, el liderazgo, el empoderamiento y la sinergia.

A continuación, se presenta una lista con los principales aspectos extraídos de la encuesta sobre la percepción del grupo participante en la actividad recreativa: 


\section{Figura 6}

Tipo de habilidades desarrolladas por el estudiantado

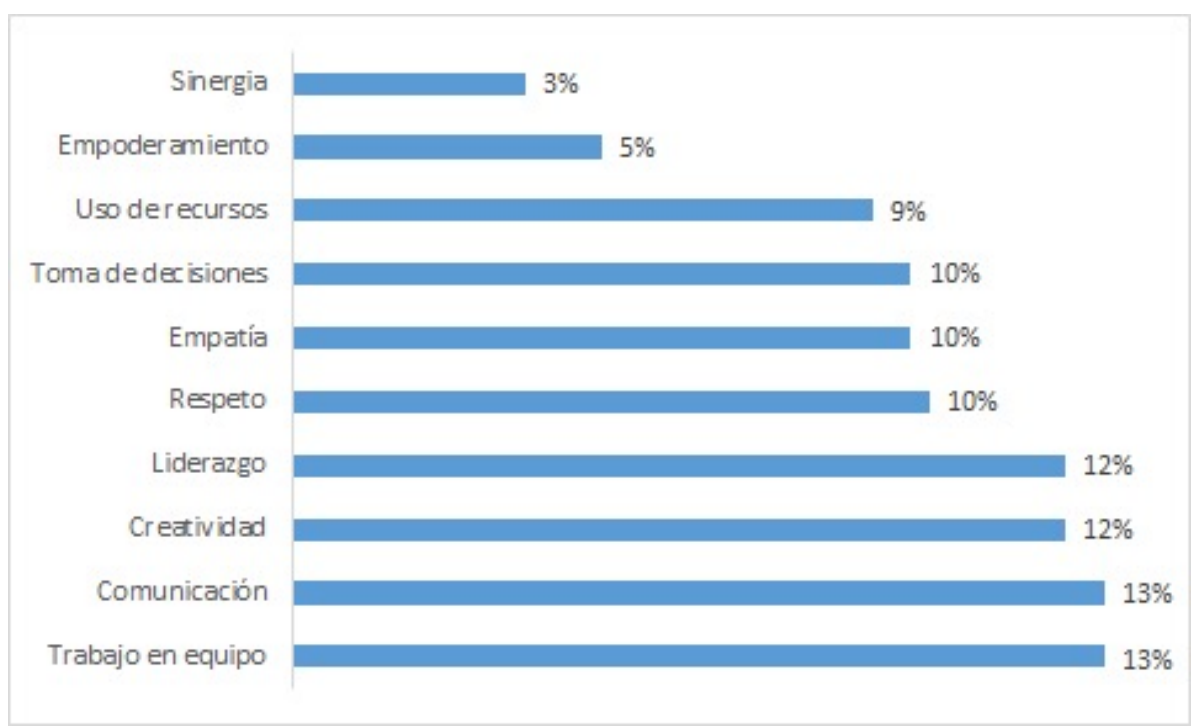

Nota: Elaboración propia

En relación con la opinión presentada por los y las representantes de las empresas, las personas estudiantes también opinan que el trabajo en equipo sigue liderando el grupo de habilidades necesarias para el éxito de la actividad, seguida por la comunicación con un $13 \%$ y la creatividad y el liderazgo con un $12 \%$. Las habilidades con menor puntaje son el respeto, la empatía, la toma de decisiones, el uso de recursos, el empoderamiento y la sinergia.

A continuación, se presenta una lista con los aspectos más relevantes sobre la percepción del grupo participante en la actividad recreativa:

- Buena respuesta de parte de la población que recibió la actividad.

- Aprendizaje en el proceso, sin embargo, faltó considerar algunos aspectos.

- Al inicio fue difícil empezar con la actividad.

- El grupo fue muy participativo y dinámico. 
URL: http://www.revistas.una.ac.cr/index.php/dialogo/index

CORREO ELECTRÓNICO: universidadendialogo@una.cr

DOI: http://doi.org/10.15359/udre.11-1.5

- Altamente provechosa, además de que aportó conocimiento sobre el trato hacia la población.

- Se llevaba una propuesta y costó realizarla.

- Fue una actividad con muchos beneficios.

- Desinterés al inicio, ya que éramos jóvenes con jóvenes; después mostraron interés.

- Excelente, la colaboración del grupo favorecía las actividades, a pesar de que la edad era un factor preocupante.

- Fue un grupo perfecto, participativo, entregado y con muchas ganas de realizar las actividades.

\section{Figura 7}

Percepción del grupo participante en la actividad recreativa
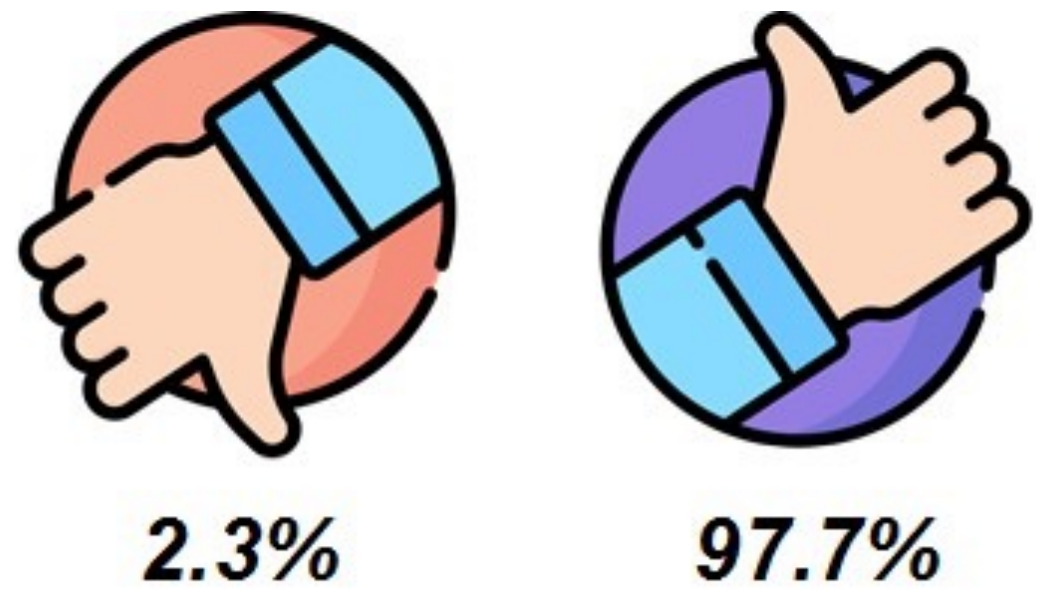

Nota. Iconos de https://www.flaticon.com/. Datos de elaboración propia

A partir de la figura anterior, y según los comentarios emitidos por la población estudiantil, es de gran relevancia que los cursos universitarios desarrollen diferentes actividades donde los y las jóvenes tengan un acercamiento con diferentes poblaciones, que exista una sensibilización de sus necesidades y se lleven a la acción los conocimientos adquiridos. 


\section{Figura 8}

Impacto de la actividad en las comunidades

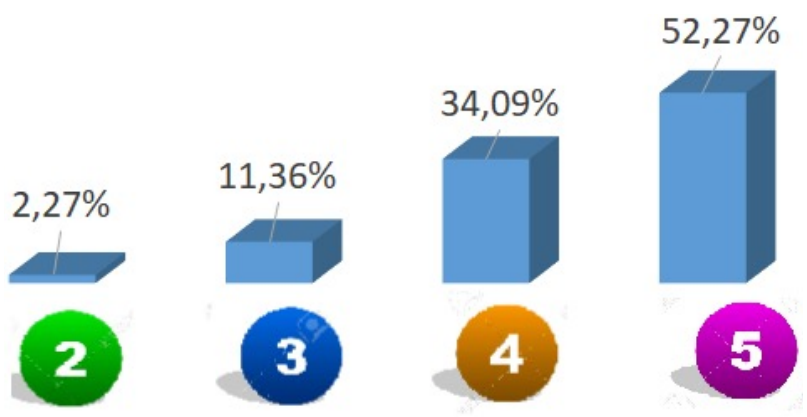

Nota. Elaboración propia

En este aspecto, se solicitó al estudiantado evaluar de 1 a 5 el nivel de impacto de la actividad efectuada en la comunidad, donde 5 es la calificación más alta con un $52,27 \%$, seguida de 4 , que obtuvo un $34,09 \%$. Ambas incluyen más del $85 \%$ de las personas encuestadas, con la clara opinión de que la actividad efectuada le proporcionó a su población un aprendizaje significativo.

Se trabajó en la necesidad de movimiento físico y de educación sobre el cuidado de la salud, así como también en la posibilidad de que ellas, como personas universitarias jóvenes, puedan generar un impacto positivo de cambio en su entorno y en las personas de sus comunidades.

\section{Resultados}

Las poblaciones beneficiadas con esta acción social abarcan 10 instituciones públicas y privadas de la provincia de Alajuela; principalmente de los cantones de Atenas, Grecia y Central, para un total de 114 personas beneficiadas con las propuestas.

Según la percepción de estas instituciones, este tipo de propuestas originan el desarrollo de habilidades, tales como: trabajo en equipo, respeto y empatía, las cuales, según indican, tienen gran relevancia para incursionar en el sector empresarial.

La percepción del grupo participante en la actividad fue muy positiva, resaltando aspectos tales como satisfacción, desarrollo de habilidades, comunicación efectiva y liderazgo. 
La percepción estudiantil respecto a la actividad destaca el aprendizaje significativo y la sensibilización sobre la importancia de la actividad física y la experiencia de la interacción con diferentes poblaciones. Destaca, además, la posibilidad de liberar estrés y energía a través de actividades entretenidas que promuevan la comunicación y la necesidad de disminuir el uso de la tecnología.

\section{Planteamiento para la gestión de la extensión}

Como resultado del trabajo efectuado, se presenta una propuesta para la gestión de la extensión en el ámbito institucional que incluye varias etapas:

\section{Primera etapa: normalización}

Esta etapa consiste en la normalización institucional de la oferta académica para todas las carreras. Es de interés institucional que, desde las vicerrectorías de extensión de cada una de las universidades, se normalice la apertura permanente de cursos optativos que promuevan la actividad física y la recreación como una estrategia para el impulso de la acción social y el desarrollo de habilidades blandas en la vida de nuevos y nuevas profesionales.

Esta acción tendrá como indicadores de logro el número de actividades desarrolladas por la población estudiantil en las comunidades, así como el número de instituciones y de personas beneficiadas con estas actividades.

\section{Segunda etapa: compartir la experiencia}

Esta etapa consiste en compartir la experiencia de los primeros grupos participantes con las personas (estudiantado y cuerpo académico) de nuevo ingreso, con las que se puede generar un alto compromiso, y para lograrlo se propone lo siguiente:

- Garantizar que, como parte de los procesos de inducción a la vida universitaria, tanto para los sectores estudiantiles como para los académicos, se conozca la experiencia de las personas que han participado en actividades con comunidades, de manera que se logre influir en las nuevas generaciones universitarias respecto al compromiso con los entornos más cercanos. 
- La conformación de una comisión de estudiantes que promocionen, motiven y expongan sus vivencias y experiencias, con el fin de transmitir de una forma más natural y con su mismo lenguaje, que aporte desde su quehacer universitario. Se puede realizar en las comunidades cercanas a los recintos universitarios.

\section{Tercera etapa: permanencia}

Con esta etapa se espera que las vicerrectorías de extensión de cada universidad instruyan a nivel de facultades, centros y sedes en relación con la permanencia de este tipo de acciones de extensión y de divulgación de los resultados como una parte del proceso de formación universitaria.

- Mantener de forma consistente la misión, los principios, los fines y los valores de las universidades, así como las metodologías de aprendizaje que aborden necesidades de diferentes grupos poblacionales, con los cuales el estudiantado pueda generar un vínculo con un impacto social a través del aprendizaje. De forma paralela, esto trae resultados positivos en el desarrollo de habilidades blandas y experiencias significativas que integran la teoría con la práctica mediante la ejecución de distintas actividades.

- El eje central de esta propuesta es implementar cursos optativos en las carreras universitarias, que permitan desarrollar acciones y actividades donde se realice un abordaje de contenidos actitudinales que le permitan a la población estudiantil la sensibilización y puesta en práctica de diferentes habilidades que la hagan consciente de las necesidades de los grupos vulnerables de la sociedad y que, a su vez, la lleve a la acción. Mediante prácticas que se desarrollen de forma natural e incluso como parte de su quehacer en el proceso de aprendizaje en la universidad.

\section{Cuarta etapa: aplicación en las carreras}

La conformación de equipos de personas docentes y estudiantes que promuevan los talleres desde los cursos optativos es muy importante para definir los siguientes aspectos:

- Grupos poblacionales que se van a atender.

- Los cursos optativos que incorporarán estas acciones. 
- Planificación de objetivos y acciones alineados con la misión, la visión y los valores institucionales

- Recursos necesarios

- Herramientas de control para medir los resultados de los objetivos e indicadores planteados

\section{Quinta etapa: mejora}

La identificación de los aspectos críticos de mejora se aplica en esta etapa. Esto se deriva de las herramientas de control aplicadas y la comparación entre los objetivos planteados y los objetivos obtenidos.

Es necesario que se unifiquen los criterios de los actores involucrados en el proceso y que se establezcan acciones de mejora para los próximos talleres de los semestres posteriores, que sea un proceso que se alimente siempre de las experiencias, que busque la mejora continua y que permita dejar una huella en todas las personas que forman parte de estas actividades.

\section{Conclusiones}

El acercamiento del estudiantado a las distintas poblaciones locales aporta un valor adicional en su formación integral, esto por medio de los vínculos que se generan en la interacción con los grupos externos a la universidad.

Esta es una forma de atender el llamado que la sociedad costarricense expresa respecto a la misión que deben cumplir las instituciones de educación superior, las cuales deben alinear el proceso de aprendizaje con el compromiso social, el conocimiento transformador, inclusivo y humanista; parte de los principios y valores de las universidades.

En los resultados obtenidos en la investigación se visualizan detalles notables sobre las habilidades que deben desplegar las personas estudiantes, considerando que no se trata solamente de un acercamiento con los grupos poblacionales, sino que la actividad que se ejecuta debe tener un impacto en dos vías: primero con las personas que reciben el beneficio y en segundo lugar con ellas mismas, que asumen en su totalidad la responsabilidad de la actividad.

De esta manera, se construye a partir de pequeños modelos que serán la base para que, en el corto plazo, se produzca una réplica de esta acción con 
la implementación en otros cursos en las universidades públicas; esto a su vez permite vincular el proceso de aprendizaje con el compromiso social, el conocimiento transformador, inclusivo y humanista; parte de los principios y valores de las instituciones de educación superior.

La percepción estudiantil respecto a las actividades generadas destaca el aprendizaje significativo y la sensibilización sobre la importancia de la actividad física y la experiencia de la interacción con diferentes poblaciones. El estudiantado enfatiza, además, la posibilidad de liberar estrés y energía a través de actividades que son entretenidas, que promueven la comunicación y disminuyen el uso de la tecnología.

El aporte de esta ponencia está dirigido a evidenciar la necesidad de considerar en los programas de los cursos y en los planes de estudio de las carreras universitarias la posibilidad de ejecutar acciones donde se integre a las comunidades sin limitar la aplicación de los contenidos curriculares de los cursos. Esto permite la atención inmediata de necesidades de diferentes grupos poblacionales con los cuales el estudiantado genera un vínculo con un impacto social a través del aprendizaje y un beneficio en su formación profesional.

\section{Referencias}

Campos, G., Covarrubias, N. y Lule, E. (2012). La observación, un método para el estudio de la realidad. Revista Xihmai, (7),13. https://dialnet. unirioja.es/servlet/articulo? codigo $=3979972$

Chacón, M. L. (2014). Gestión educativa del siglo XXI: bajo el paradigma emergente de la complejidad. Omnia, 20(2), 150-161. http://www. redalyc.org/pdf/737/73735396006.pdf

González, B. y Orozco, R. (2013). La capacitación del sector administrativo en las universidades estatales de Costa Rica. Universidad Estatal a Distancia, Costa Rica. https://core.ac.uk/download/pdf/67707751.pdf

Guerrero-Ramos, D., Jiménez-Torres, M. y López-Sánchez, M. (2014). Escuela saludable versus protectora de la salud (salugénica). Educar, 50(2), 323-338. https://www.redalyc.org/pdf/3421/Resumenes/ Resumen_342132463008 1.pdf 
Moscoso, Sánchez, Martin y Pedrajas. (2014). ¿Qué significa ser activo en una sociedad sedentaria? Paradojas de los estilos de vida y el ocio en la juventud española. Empiria. Revista de Metodología de Ciencias Sociales, (30), 77-108. https://doi.org/10.5944/empiria.30.2015.13886

Neumann, A. (2015). Talleres que potencian las habilidades socioemocionales y el desempeño futuro. El Mercurio. http://diario.elmercurio.com/ detalle/index.asp?id $=\{$ e3f67cfc-380e-46ef-bf0a-04231f5ea29e $\}$

Pérez,A.(2013). Enfoques de la gestión escolar: una aproximación desde el contexto latinoamericano. Educación, 17(2), 357-369. 10.5294/edu.2014.17.2.9

Ramírez, C. A., Calderón, H. G. y Castaño-Duque, G. A. (2015). Enfoques administrativos presentes en establecimientos educativos. Revista Mexicana de Investigación Educativa, 20(66), 911-940.https://searchebscohost-com.una.idm.oclc.org/login.aspx?direct=true\&AuthType $=$ ip ,uid,url\&db=ehh\&AN=109100905\&lang=es\&site=ehost-live

Ramos,M.(2014).Técnicasymétodosderecoleccióndedatos.https://es.slideshare. net/JoseMendozaCastillo/12-instrumentos-de-recoleccin-de-datos.

Salas, O. (2017). Mayoría de la población en Costa Rica es sedentaria. Universidad de Costa Rica. https://www.ucr.ac.cr/noticias/2017/01/23/ mayoria-de-la-poblacion-en-costa-rica-es-sedentaria.html

Santamaría, M. y Martínez, (2018). Las escuelas promotoras de salud: un reto por descubrir (trabajo final de grado). Facultad de Educación de Palencia, Universidad de Valladolid. https:/uvadoc.uva.es/ bitstream/10324/29324/1/TFG-L1922.pdf

Vargas. M. (2017). Relación entre el deporte y la motivación laboral en el fortalecimiento de las habilidades blandas dentro de los colaboradores del Departamento de Operaciones Financieras y Riesgo de una entidad de servicios compartidos. Universidad Estatal a Distancia. San José, Costa Rica. http://aleph.uned.ac.cr/exlibris/aleph/a21 1/apache media/ D532U1564X2CLAY3D7DJ7KKVCY4SFB.pdf

Werther, W. y Davis, K. (2014). Administración de recursos humanos. Gestión del capital humano ( $7^{\mathrm{a}}$ ed.). México: Editorial McGraw-Hill Education. 